Article

\title{
Certain Concepts in Intuitionistic Neutrosophic Graph Structures
}

\author{
Muhammad Akram * (D) and Muzzamal Sitara \\ Department of Mathematics, University of the Punjab, Quaid-e-Azam Campus, 54590 Lahore, Pakistan; \\ muzzamalsitara@gmail.com \\ * Correspondence: m.akram@pucit.edu.pk
}

Received: 2 November 2017; Accepted: 19 November 2017; Published: 25 November 2017

\begin{abstract}
A graph structure is a generalization of simple graphs. Graph structures are very useful tools for the study of different domains of computational intelligence and computer science. In this research paper, we introduce certain notions of intuitionistic neutrosophic graph structures. We illustrate these notions by several examples. We investigate some related properties of intuitionistic neutrosophic graph structures. We also present an application of intuitionistic neutrosophic graph structures.
\end{abstract}

Keywords: graph structure; intuitionistic neutrosophic graph structure; $\psi$-complement

MSC: 03E72; 05C72; 05C78; 05C99

\section{Introduction}

Fuzzy graph models are advantageous mathematical tools for dealing with combinatorial problems of various domains including operations research, optimization, social science, algebra, computer science, environmental science and topology. Fuzzy graphical models are obviously better than graphical models due to natural existence of vagueness and ambiguity. Initially, we needed fuzzy set theory to cope with many complex phenomenons having incomplete information. Fuzzy set theory [1] is a very strong mathematical tool for solving approximate reasoning related problems. These notions describe complex phenomenons very well, which are not properly described using classical mathematics. Atanassov [2] generalized the fuzzy set theory by introducing the notion of intuitionistic fuzzy sets. The intuitionistic fuzzy sets have more describing possibilities as compared to fuzzy sets. An intuitionistic fuzzy set is inventive and more useful due to the existence of non-membership degree. In many situations like information fusion, indeterminacy is explicitly quantified. Smarandache [3] introduced the concept of neutrosophic sets, and he combined the tricomponent logic, non-standard analysis, and philosophy. It is a branch of philosophy which studies the origin, nature and scope of neutralities as well as their interactions with different ideational spectra. Three independent components of neutrosophic set are: truth value, indeterminacy value and falsity value [3]. For convenient use of neutrosophic sets in real-life phenomena, Wang et al. [4] proposed single valued neutrosophic sets, which is a generalization of intuitionistic fuzzy sets [2] and has three independent components having values in a standard unit interval [0,1]. Ye [5-8] proposed several multi criteria decision-making methods based on neutrosophic sets. Bhowmik and Pal $[9,10]$ introduced the notion of intuitionistic neutrosophic sets.

Kauffman [11] introduced fuzzy graphs on the basis of Zadeh's fuzzy relations [12]. Rosenfeld [13] discussed fuzzy analogue of many graph-theoretic notions. Later on, Bhattacharya [14] gave 
some remarks on fuzzy graphs. The complement of a fuzzy graph was defined by Sunitha and Vijayakumar [15]. Bhutani and Rosenfeld studied the notion of $M$-strong fuzzy graphs and their properties in [16]. Parvathi et al. defined operations on intuitionistic fuzzy graphs in [17]. Akram and Shahzadi [18] introduced neutrosophic soft graphs with applications. Dinesh and Ramakrishnan [19] introduced the notion of fuzzy graph structures and discussed some related properties. Akram and Akmal [20] introduced the concept of bipolar fuzzy graph structures. Recently, Akram and Sitara [21] introduced the concept of intuitionistic neutrosophic graph structures. Several notions' graph structures have been studied by the same authors in [22-27]. In this research paper, we introduce certain notions of intuitionistic neutrosophic graph structures and illustrate these notions by examples. We also present an application of intuitionistic neutrosophic graph structures in decision-making. For other notations and applications, readers are referred to [28-45] .

\section{Intuitionistic Neutrosophic Graph Structures}

Sampathkumar [46] introduced the graph structure, which is a generalization of an undirected graph and is quite useful in studying some structures like graphs, signed graphs, labeled graphs and edge colored graphs.

Definition 1. [46] A graph structure $G=\left(V, R_{1}, \ldots, R_{r}\right)$ consists of a non-empty set $V$ together with relations $R_{1}, R_{2}, \ldots, R_{r}$ on $V$, which are mutually disjoint such that each $R_{h}, 1 \leq h \leq r$ is symmetric and irreflexive.

One can represent a graph structure $G=\left(V, R_{1}, \ldots, R_{r}\right)$ in the plane, just like a graph where each edge is labeled as $R_{h}, 1 \leq h \leq r$.

Definition 2. [3] An ordered triple $<T_{N}, I_{N}, F_{N}>$ in $] 0^{-}, 1^{+}[$in the universe of discourse $V$ is called neutrosophic set, where $\left.T_{N}, I_{N}, F_{N}: V \rightarrow\right] 0^{-}, 1^{+}[$, and their sum is without any restriction.

Definition 3. [4] An ordered triple $<T_{N}, I_{N}, F_{N}>$ in $[0,1]$ in a universe of discourse $V$ is called single-valued neutrosophic set, where $T_{N}, I_{N}, F_{N}: V \rightarrow[0,1]$, and their sum is restricted between 0 and 3 .

Definition 4. [47] Let $V$ be a fixed set. A generalized intuitionistic fuzzy set I of $V$ is an object having the form $I=\left\{\left(u, \mu_{I}(u), v_{I}(u)\right) \mid u \in V\right\}$, where the functions $\mu_{I}(u): \rightarrow[0,1]$ and $v_{I}(u): \rightarrow[0,1]$ define the degree of membership and degree of nonmembership of an element $u \in V$, respectively, such that

$$
\min \left\{\mu_{I}(u), v_{I}(u)\right\} \leq 0.5, \text { for all } u \in V .
$$

Definition 5. [9,10] An intuitionistic neutrosophic set can be stated as a set having the form $I=$ $\left\{T_{I}(u), I_{I}(u), F_{I}(u): u \in V\right\}$, where

$$
\begin{aligned}
& \min \left\{T_{I}(u), I_{I}(u)\right\} \leq 0.5, \\
& \min \left\{F_{I}(u), I_{I}(u)\right\} \leq 0.5, \\
& \min \left\{T_{I}(u), F_{I}(u)\right\} \leq 0.5,
\end{aligned}
$$

and $0 \leq T_{I}(u)+I_{I}(u)+F_{I}(u) \leq 2$.

Definition 6. Let $\breve{G}=\left(P, P_{1}, P_{2}, \ldots, P_{r}\right)$ be a graph structure(GS), and then $\breve{G}_{i}=\left(O, O_{1}, O_{2}\right.$ and $\left.\ldots, O_{r}\right)$ is called an intuitionistic neutrosophic graph structure (INGS), if $O=\left\langle k, T(k), I(k), F(k)>\right.$ and $O_{h}=$ $<(k, l), T_{h}(k, l), I_{h}(k, l), F_{h}(k, l)>$ are intuitionistic neutrosophic sets on $P$ and $P_{h}$, respectively, such that

1. $\quad T_{h}(k, l) \leq T(k) \wedge T(l), \quad I_{h}(k, l) \leq I(k) \wedge I(l), \quad F_{h}(k, l) \leq F(k) \vee F(l) ;$

2. $\quad T_{h}(k, l) \wedge I_{h}(k, l) \leq 0.5, \quad T_{h}(k, l) \wedge F_{h}(k, l) \leq 0.5, \quad I_{h}(k, l) \wedge F_{h}(k, l) \leq 0.5 ;$ 
3. $0 \leq T_{h}(k, l)+I_{h}(k, l)+F_{h}(k, l) \leq 2, \quad \forall(k, l) \in O_{h}, h=1,2, \ldots, r$,

where $O$ is an underlying vertex set of $\check{G}_{i}$ and $O_{h}(h=1,2, \ldots, r)$ are underlying $h$-edge sets of $\breve{G}_{i}$.

Example 1. Consider a $G S \breve{G}=\left(P, P_{1}, P_{2}\right)$ such that $O, O_{1}, O_{2}$ are IN subsets of $P, P_{1}, P_{2}$, respectively, where

$$
\begin{aligned}
P & =\left\{k_{1}, k_{2}, k_{3}, k_{4}, k_{5}, k_{6}, k_{7}, k_{8}\right\}, \\
P_{1} & =\left\{k_{1} k_{2}, k_{3} k_{4}, k_{5} k_{6}, k_{3} k_{7}, k_{6} k_{8}\right\}, \\
P_{2} & =\left\{k_{2} k_{3}, k_{4} k_{5}, k_{1} k_{6}, k_{5} k_{7}, k_{2} k_{8}\right\} .
\end{aligned}
$$

Through direct calculations, it is easy to show that $\breve{G}_{i}=\left(O, O_{1}, O_{2}\right)$ is an INGS of $\breve{G}$ as represented in Figure 1.

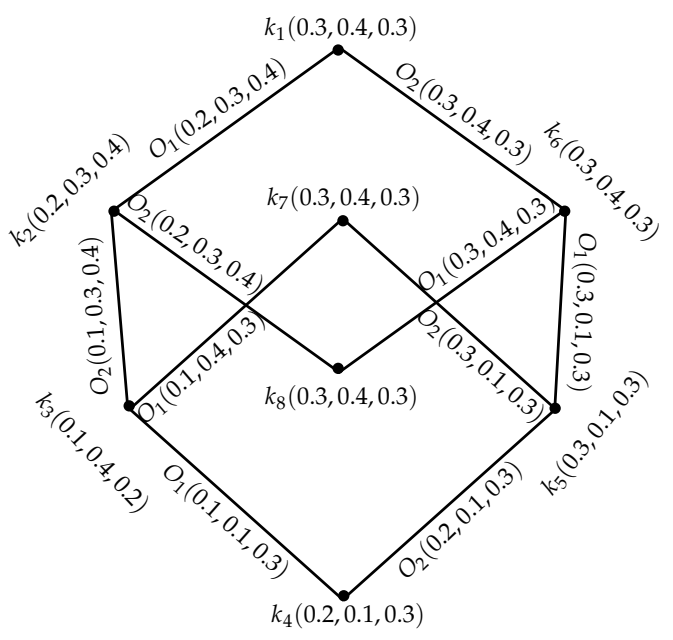

Figure 1. An intuitionistic neutrosophic graph structure.

Definition 7. Let $\check{G}_{i}=\left(O, O_{1}, O_{2}, \ldots, O_{r}\right)$ be an INGS of $\check{G}$. If $\check{H}_{i}=\left(O^{\prime}, O_{1}^{\prime}, O_{2}^{\prime}, \ldots, O_{r}^{\prime}\right)$ is an INGS of $\check{G}$ such that

$$
\begin{gathered}
T^{\prime}(k) \leq T(k), I^{\prime}(k) \leq I(k), F^{\prime}(k) \geq F(k) \forall k \in P, \\
T_{h}^{\prime}(k, l) \leq T_{h}(k, l), I_{h}^{\prime}(k, l) \leq I_{h}(k, l), F_{h}^{\prime}(k, l) \geq F_{h}(k, l), \forall(k, l) \in P_{h}, h=1,2, \ldots, r .
\end{gathered}
$$

Then, $\check{H}_{i}$ is said to be an intuitionistic neutrosophic (IN) subgraph structure of INGS $\check{G}_{i}$.

Example 2. Consider an INGS $\check{H}_{i}=\left(O^{\prime}, O_{1}^{\prime}, O_{2}^{\prime}\right)$ of $G S \breve{G}=\left(P, P_{1}, P_{2}\right)$ as represented in Figure 2. Through routine calculations, it can be easily shown that $\check{H}_{i}$ is an IN subgraph structure of INGS $\breve{G}_{i}$. 


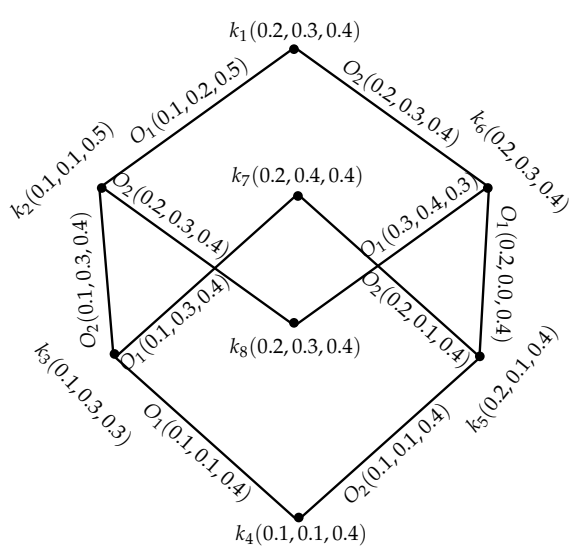

Figure 2. IN subgraph structure.

Definition 8. An INGS $\check{H}_{i}=\left(O^{\prime}, O_{1}^{\prime}, O_{2}^{\prime}, \ldots, O_{r}^{\prime}\right)$ is called an IN induced-subgraph structure of $\check{G}_{i}$ by $Q \subseteq P$ if

$$
\begin{gathered}
T^{\prime}(k)=T(k), I^{\prime}(k)=I(k), F^{\prime}(k)=F(k), \forall k \in Q, \\
T_{h}^{\prime}(k, l)=T_{h}(k, l), I_{h}^{\prime}(k, l)=I_{h}(k, l), F_{h}^{\prime}(k, l)=F_{h}(k, l), \forall k, l \in Q, h=1,2, \ldots, r .
\end{gathered}
$$

Example 3. The INGS in the given Figure 3 is an IN induced-subgraph structure of an INGS in Figure 1.

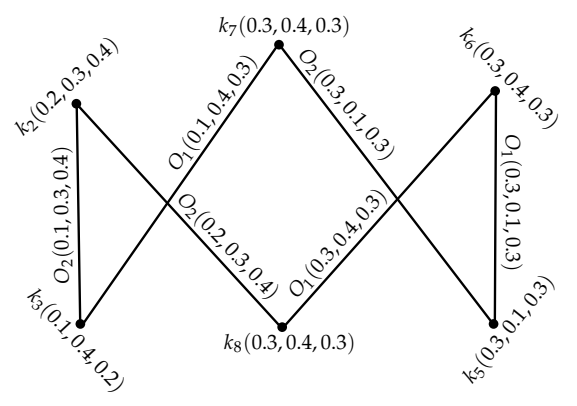

Figure 3. An IN induced-subgraph structure.

Definition 9. An INGS $\breve{H}_{i}=\left(O^{\prime}, O_{1}^{\prime}, O_{2}^{\prime}, \ldots, O_{r}^{\prime}\right)$ is said to be a IN spanning-subgraph structure of $\check{G}_{i}$ if $O^{\prime}=$ $O$ and

$$
T_{h}^{\prime}(k, l) \leq T_{h}(k, l), I_{h}^{\prime}(k, l) \leq I_{h}(k, l), F_{h}^{\prime}(k, l) \geq F_{h}(k, l), h=1,2, \ldots, r .
$$

Example 4. An INGS shown in Figure 4 is an IN spanning-subgraph structure of an INGS in Figure 1. 


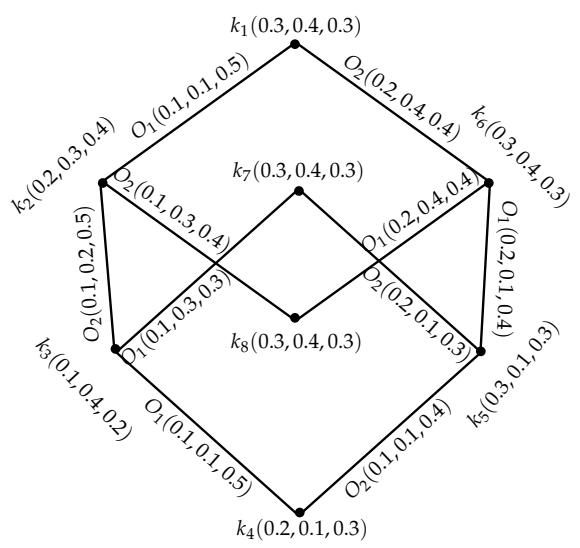

Figure 4. An IN spanning-subgraph structure.

Definition 10. Let $\breve{G}_{i}=\left(O, O_{1}, O_{2}, \ldots, O_{r}\right)$ be an INGS. Then, $k l \in P_{h}$ is named as a IN $O_{h}$-edge or shortly $O_{h}$-edge, if $T_{h}(k, l)>0$ or $I_{h}(k, l)>0$ or $F_{h}(k, l)>0$ or all these conditions are satisfied. As a result, support of $O_{h}$ is:

$$
\operatorname{supp}\left(O_{h}\right)=\left\{k l \in O_{h}: T_{h}(k, l)>0\right\} \cup\left\{k l \in O_{h}: I_{h}(k, l)>0\right\} \cup\left\{k l \in O_{h}: F_{h}(k, l)>0\right\},
$$

$h=1,2, \ldots, r$

Definition 11. $O_{h}$-path in an INGS $\check{G}_{i}=\left(O, O_{1}, O_{2}, \ldots, O_{r}\right)$ is a sequence $k_{1}, k_{2}, \ldots, k_{r}$ of distinct vertices (except $k_{r}=k_{1}$ ) in $P$, such that $k_{h-1} k_{h}$ is an IN $O_{h}$-edge $\forall h=2, \ldots, r$.

Definition 12. An INGS $\breve{G}_{i}=\left(O, O_{1}, O_{2}, \ldots, O_{r}\right)$ is $O_{h}$-strong for any $h \in\{1,2, \ldots, r\}$ if

$$
T_{h}(k, l)=\min \{T(k), T(l)\}, I_{h}(k, l)=\min \{I(k), I(l)\}, F_{h}(k, l)=\max \{F(k), F(l)\},
$$

$\forall k l \in \operatorname{supp}\left(O_{h}\right)$. If $\check{G}_{i}$ is $O_{h}$-strong for all $h \in\{1,2, \ldots, r\}$, then $\check{G}_{i}$ is a strong INGS.

Example 5. Consider an INGS $\check{G}_{i}=\left(O, O_{1}, O_{2}\right)$ as represented in Figure 5. Then, $\breve{G}_{i}$ is strong INGS, as it is $\mathrm{O}_{1}-$ and $\mathrm{O}_{2}-$ strong.

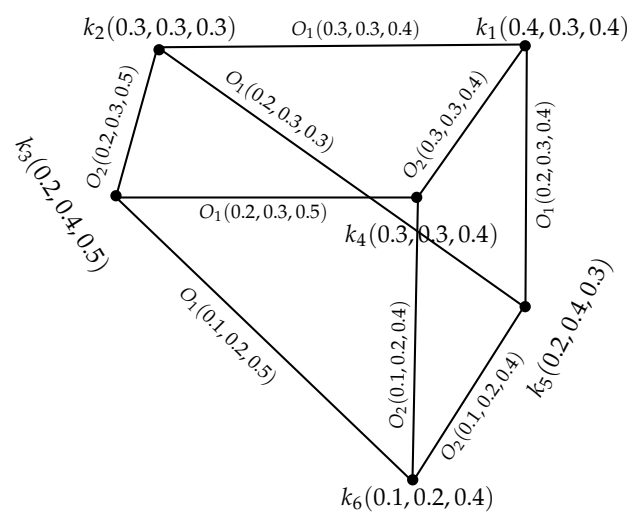

Figure 5. A strong INGS.

Definition 13. An INGS $\breve{G}_{i}=\left(O, O_{1}, O_{2}, \ldots, O_{r}\right)$ is a complete INGS, if

1. $\breve{G}_{i}$ is strong INGS. 
2. $\operatorname{supp}\left(O_{h}\right) \neq \varnothing$, for all $h=1,2, \ldots, r$.

3. For all $k, l \in P, k l$ is a $O_{h}-$ edge for some $h$.

Example 6. Let $\breve{G}_{i}=\left(O, O_{1}, O_{2}\right)$ be an INGS of GS $\breve{G}=\left(P, P_{1}, P_{2}\right)$, such that

$$
\begin{gathered}
P=\left\{k_{1}, k_{2}, k_{3}, k_{4}, k_{5}, k_{6}\right\}, \\
P_{1}=\left\{k_{1} k_{6}, k_{1} k_{2}, k_{2} k_{4}, k_{2} k_{5}, k_{2} k_{6}, k_{1} k_{6}\right\}, \\
P_{2}=\left\{k_{2} k_{6}, k_{4} k_{3}, k_{5} k_{6}, k_{1} k_{4}\right\}, \\
P_{3}=\left\{k_{1} k_{5}, k_{5} k_{3}, k_{2} k_{3}, k_{1} k_{3}, k_{4} k_{6}\right\} .
\end{gathered}
$$

By means of direct calculations, it is easy to show that $\check{G}_{i}$ is strong INGS.

Moreover, $\operatorname{supp}\left(O_{1}\right) \neq \varnothing, \operatorname{supp}\left(O_{2}\right) \neq \varnothing, \operatorname{supp}\left(O_{3}\right) \neq \varnothing$, and every pair $k_{h} k_{q}$ of vertices of $P$, is $O_{1}$-edge or $\mathrm{O}_{2}$-edge or an $\mathrm{O}_{3}$-edge. Hence, $\breve{G}_{i}$ is a complete INGS, that is, $\mathrm{O}_{1} \mathrm{O}_{2} \mathrm{O}_{3}$-complete INGS.

Definition 14. Let $\check{G}_{i}=\left(O, O_{1}, O_{2}, \ldots, O_{r}\right)$ be an INGS. The truth strength T.P. $P_{\mathrm{O}^{\prime}}$ falsity strength F.P. $P_{\mathrm{O}_{H^{\prime}}}$ and indeterminacy strength I. $P_{O_{h}}$ of an $O_{h}$-path, $P_{O_{h}}=k_{1}, k_{2}, \ldots, k_{n}$ is defined as:

$$
\begin{aligned}
& \text { T. } P_{O_{h}}=\bigwedge_{i=2}^{n}\left[T_{O_{h}}^{P}\left(k_{i-1} k_{i}\right)\right], \\
& \text { I.P } P_{O_{h}}=\bigwedge_{i=2}^{n}\left[I_{O_{h}}^{P}\left(k_{i-1} k_{i}\right)\right], \\
& \text { F.P } P_{O_{h}}=\bigvee_{i=2}^{n}\left[F_{O_{h}}^{P}\left(k_{i-1} k_{i}\right)\right] .
\end{aligned}
$$

Example 7. Consider an INGS $\breve{G}_{i}=\left(O, O_{1}, O_{2}, O_{3}\right)$ as in Figure 6. We found an $O_{1}$-path $P_{O_{1}}=k_{2}, k_{1}, k_{6}$. So, T.P. $P_{O_{1}}=0.2$, I. $P_{\mathrm{O}_{1}}=0.1$ and F. $P_{\mathrm{O}_{2}}=0.5$.

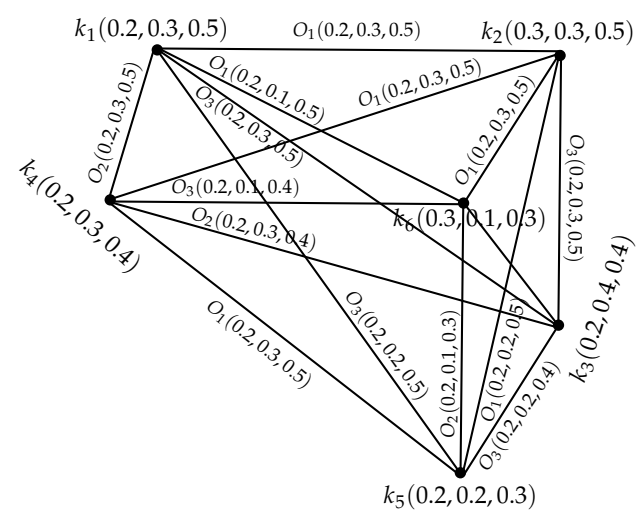

Figure 6. A complete INGS.

Definition 15. Let $\breve{G}_{i}=\left(O, O_{1}, O_{2}, \ldots, O_{r}\right)$ be an INGS. Then,

- $\quad O_{h}$-strength of connectedness of truth between $k$ and $l$ is defined as: $T_{O_{h}}^{\infty}(k l)=\bigvee_{i \geq 1}\left\{T_{O_{h}}^{i}(k l)\right\}$, such that $T_{O_{h}}^{i}(k l)=\left(T_{O_{h}}^{i-1} \circ T_{O_{h}}^{1}\right)(k l)$ for $i \geq 2$ and $T_{O_{h}}^{2}(k l)=\left(T_{O_{h}}^{1} \circ T_{O_{h}}^{1}\right)(k l)=\bigvee_{y}\left(T_{O_{h}}^{1}(k y) \wedge T_{O_{h}}^{1}\right)(y l)$.

- $\quad O_{h}$-strength of connectedness of indeterminacy between $k$ and $l$ is defined as: $I_{O_{h}}^{\infty}(k l)=\bigvee_{i \geq 1}\left\{I_{O_{h}}^{i}(k l)\right\}$, such that $I_{O_{h}}^{i}(k l)=\left(I_{O_{h}}^{i-1} \circ I_{O_{h}}^{1}\right)(k l)$ for $i \geq 2$ and $I_{O_{h}}^{2}(k l)=\left(I_{O_{h}}^{1} \circ I_{O_{h}}^{1}\right)(k l)=\bigvee_{y}\left(I_{O_{h}}^{1}(k y) \wedge I_{O_{i}}^{1}\right)(y l)$. 
- $O_{h}$-strength of connectedness of falsity between $k$ and $l$ is defined as: $F_{O_{h}}^{\infty}(k l)=\bigwedge_{i \geq 1}\left\{F_{O_{h}}^{i}(k l)\right\}$, such that $F_{O_{h}}^{i}(k l)=\left(F_{O_{h}}^{i-1} \circ F_{O_{h}}^{1}\right)(k l)$ for $i \geq 2$ and $F_{Q_{h}}^{2}(k l)=\left(F_{O_{h}}^{1} \circ F_{O_{h}}^{1}\right)(k l)=\bigwedge_{y}\left(F_{O_{h}}^{1}(k y) \vee F_{O_{h}}^{1}\right)(y l)$.

Definition 16. An INGS $\check{G}_{i}=\left(O, O_{1}, O_{2}, \ldots, O_{r}\right)$ is called an $O_{h}$-cycle if $\left(\operatorname{supp}(O), \operatorname{supp}\left(O_{1}\right)\right.$, $\left.\operatorname{supp}\left(\mathrm{O}_{2}\right), \ldots, \operatorname{supp}\left(\mathrm{O}_{r}\right)\right)$ is an $\mathrm{O}_{h}-$ cycle.

Definition 17. An INGS $\breve{G}_{i}=\left(O, O_{1}, O_{2}, \ldots, O_{r}\right)$ is an IN fuzzy $O_{h}$-cycle (for any $\left.h\right)$ if

1. $\check{G}_{i}$ is an $\mathrm{O}_{h}$-cycle.

2. There exists no unique $O_{h}$-edge $k l$ in $\breve{G}_{i}$ such that

$T_{O_{h}}(k l)=\min \left\{T_{O_{h}}(y z): y z \in P_{h}=\operatorname{supp}\left(O_{h}\right)\right\}$ or $I_{O_{h}}(k l)=\min \left\{I_{O_{h}}(y z): y z \in P_{h}=\operatorname{supp}\left(O_{h}\right)\right\}$ or $F_{O_{h}}(k l)=\max \left\{F_{O_{h}}(y z): y z \in P_{h}=\operatorname{supp}\left(O_{h}\right)\right\}$.

Example 8. Consider an INGS $\check{G}_{i}=\left(O, O_{1}, O_{2}\right)$ as in Figure 6. Then, $\breve{G}_{i}$ is an $O_{1}$-cycle and IN fuzzy $O_{1}-$ cycle, since $\left(\operatorname{supp}(O), \operatorname{supp}\left(O_{1}\right), \operatorname{supp}\left(O_{2}\right)\right)$ is an $O_{1}$-cycle and no unique $O_{1}$-edge $k l$ satisfies the condition: $T_{O_{h}}(k l)=\min \left\{T_{O_{h}}(y z): y z \in P_{h}=\operatorname{supp}\left(O_{h}\right)\right\}$ or $I_{O_{h}}(k l)=\min \left\{I_{O_{h}}(y z): y z \in P_{h}=\operatorname{supp}\left(O_{h}\right)\right\}$ or $F_{O_{h}}(k l)=\max \left\{F_{O_{h}}(y z): y z \in P_{h}=\operatorname{supp}\left(O_{h}\right)\right\}$.

Definition 18. Let $\breve{G}_{i}=\left(O, O_{1}, O_{2}, \ldots, O_{r}\right)$ be an INGS and $k$ a vertex in $\check{G}_{i}$. Let $\left(O^{\prime}, O_{1}^{\prime}, O_{2}^{\prime}, \ldots, O_{r}^{\prime}\right)$ be an IN subgraph structure of $\breve{G}_{i}$ induced by $P \backslash\{k\}$ such that $\forall y \neq k, z \neq k$.

$T_{O^{\prime}}(k)=0=I_{O^{\prime}}(k)=F_{O^{\prime}}(k), T_{O_{h}^{\prime}}(k y)=0=I_{O_{h}^{\prime}}(k y)=F_{O_{h}^{\prime}}(k y) \forall$ edges $k y \in \check{G}_{i} ; T_{O^{\prime}}(y)=T_{O}(y)$, $I_{O^{\prime}}(y)=I_{O}(y), F_{O^{\prime}}(y)=F_{O}(y), \forall y \neq k ; T_{O_{h}^{\prime}}(y z)=T_{O_{h}}(y z), I_{O_{h}^{\prime}}(y z)=I_{O_{h}}(y z), F_{O_{h}^{\prime}}(y z)=F_{O_{h}}(y z)$.

Then, $k$ is IN fuzzy $O_{h}$ cut-vertex, for some $h$, if

$$
T_{O_{h}}^{\infty}(y z)>T_{O_{h}^{\prime}}^{\infty}(y z), I_{O_{h}}^{\infty}(y z)>I_{O_{h}^{\prime}}^{\infty}(y z)
$$

and

$$
F_{O_{h}}^{\infty}(y z)>F_{O_{h}^{\prime}}^{\infty}(y z) \text {, for some } y, z \in P \backslash\{k\} .
$$

Note that $k$ is an IN fuzzy $O_{h}-T$ cut-vertex, if $T_{O_{h}}^{\infty}(y z)>T_{O_{h}^{\prime}}^{\infty}(y z)$, IN fuzzy $O_{h}-I$ cut-vertex, if $I_{O_{h}}^{\infty}(y z)>$ $I_{O_{h}^{\prime}}^{\infty}(y z)$ and IN fuzzy $O_{h}-F$ cut-vertex, if $F_{O_{h}}^{\infty}(y z)>F_{O_{h}^{\prime}}^{\infty}(y z)$.

Example 9. Consider an INGS $\breve{G}_{i}=\left(O, O_{1}, O_{2}\right)$ as represented in Figure 7 and $\breve{G}_{h}^{\prime}=\left(O^{\prime}, O_{1}^{\prime}, O_{2}^{\prime}\right)$ is an IN subgraph structure of an INGS $\breve{G}_{i}$, and we found it by deleting the vertex $k_{2}$. The vertex $k_{2}$ is an IN fuzzy $\mathrm{O}_{1}-I$ cut-vertex, since $I_{O_{1}^{\prime}}^{\infty}\left(k_{2} k_{5}\right)=0<0.5=I_{O_{1}}^{\infty}\left(k_{2} k_{5}\right), I_{O_{1}^{\prime}}^{\infty}\left(k_{4} k_{3}\right)=0.7=I_{O_{1}}^{\infty}\left(k_{4} k_{3}\right)$ and $I_{O_{1}^{\prime}}^{\infty}\left(k_{3} k_{5}\right)=0.3<$ $0.4=I_{O_{1}}^{\infty}\left(k_{3} k_{5}\right)$.

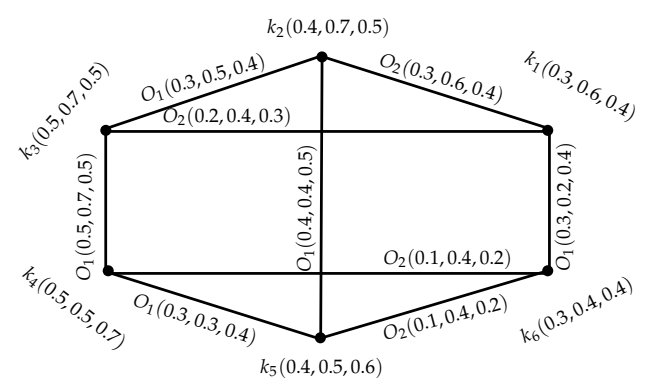

Figure 7. An INGS $\check{G}_{i}=\left(O, O_{1}, O_{2}\right)$. 
Definition 19. Let $\breve{G}_{i}=\left(O, O_{1}, O_{2}, \ldots, O_{r}\right)$ be an INGS and $k l$ an $O_{h}-e d g e$.

Let $\left(O^{\prime}, O_{1}^{\prime}, O_{2}^{\prime}, \ldots, O_{r}^{\prime}\right)$ be an IN fuzzy spanning-subgraph structure of $\check{G}_{i}$, such that $T_{O_{h}^{\prime}}(k l)=0=I_{O_{h}^{\prime}}(k l)=F_{O_{h}^{\prime}}(k l), T_{O_{h}^{\prime}}(q t)=T_{O_{h}}(q t), I_{O_{h}^{\prime}}(q t)=I_{O_{h}}(q t), F_{O_{h}^{\prime}}(q t)=F_{O_{h}}(q t)$, $\forall$ edges $q t \neq k l$.

Then, $k l$ is an IN fuzzy $O_{h}$-bridge if

$T_{O_{h}}^{\infty}(y z)>T_{O_{h}^{\prime}}^{\infty}(y z), I_{O_{h}}^{\infty}(y z)>I_{O_{h}^{\prime}}^{\infty}(y z)$ and $F_{O_{h}}^{\infty}(y z)>F_{O_{h}^{\prime}}^{\infty}(y z)$, for some $y, z \in P$.

Note that $k l$ is an IN fuzzy $O_{h}-T$ bridge if $T_{O_{h}}^{\infty}(y z)>T_{O_{h}^{\prime}}^{\infty}(y z)$, IN fuzzy $O_{h}-I$ bridge if $I_{O_{h}}^{\infty}(y z)>I_{O_{h}^{\prime}}^{\infty}(y z)$ and IN fuzzy $O_{h}-F$ bridge if $F_{O_{h}}^{\infty}(y z)>F_{O_{h}^{\prime}}^{\infty}(y z)$.

Example 10. Consider an INGS $\breve{G}_{i}=\left(O, O_{1}, O_{2}\right)$ as shown in Figure 7 and $\breve{G}_{H}^{\prime}=\left(O^{\prime \prime}, O_{1}^{\prime \prime}, O_{2}^{\prime \prime}\right)$ is IN spanning-subgraph structure of an INGS $\breve{G}_{i}$ found by the deletion of $\mathrm{O}_{1}$-edge $\left(k_{2} k_{5}\right)$. Edge $\left(k_{2} k_{5}\right)$ is an IN fuzzy $O_{1}$-bridge. As $T_{O_{1}^{\prime \prime}}^{\infty}\left(k_{2} k_{5}\right)=0.3<0.4=T_{O_{1}}^{\infty}\left(k_{2} k_{5}\right), I_{O_{1}^{\prime \prime}}^{\infty}\left(k_{2} k_{5}\right)=0.3<0.4=I_{O_{1}}^{\infty}\left(k_{2} k_{5}\right)$, $F_{O_{1}^{\prime \prime}}^{\infty}\left(k_{2} k_{5}\right)=0.4<0.5=F_{O_{1}}^{\infty}\left(k_{2} k_{5}\right)$.

Definition 20. An INGS $\breve{G}_{i}=\left(O, O_{1}, O_{2}, \ldots, O_{r}\right)$ is an $O_{h}$-tree, if $\left(\operatorname{supp}(O), \operatorname{supp}\left(O_{1}\right)\right.$, $\left.\operatorname{supp}\left(\mathrm{O}_{2}\right), \ldots, \operatorname{supp}\left(\mathrm{O}_{r}\right)\right)$ is an $\mathrm{O}_{h}-$ tree. Alternatively, $\breve{G}_{i}$ is an $\mathrm{O}_{h}$-tree, if there is a subgraph of $\check{G}_{i}$ induced by $\operatorname{supp}\left(O_{h}\right)$, which forms a tree.

Definition 21. An INGS $\check{G}_{i}=\left(O, O_{1}, O_{2}, \ldots, O_{r}\right)$ is an IN fuzzy $O_{h}$-tree if $\check{G}_{i}$ has an IN fuzzy spanning-subgraph structure $\check{H}_{i}=\left(O^{\prime \prime}, O_{1}^{\prime \prime}, O_{2}^{\prime \prime}, \ldots, O_{r}^{\prime \prime}\right)$, such that, for all $O_{h}$-edges $k l$ not in $\breve{H}_{i}$,

$\check{H}_{i}$ is an $O_{h}^{\prime \prime}$-tree, and $T_{O_{h}}(k l)<T_{O_{h}^{\prime \prime}}^{\infty}(k l), I_{O_{h}}(k l)<I_{O_{h}^{\prime \prime}}^{\infty}(k l), F_{O_{h}}(k l)<F_{O_{h}^{\prime \prime}}^{\infty}(k l)$.

In particular, $\check{G}_{i}$ is an IN fuzzy $O_{h}$-T tree if $T_{O_{h}}(k l)<T_{O_{h}^{\prime \prime}}^{\infty}(k l)$, an IN fuzzy $O_{h}$-I tree if

$I_{O_{h}}(k l)<I_{O_{h}^{\prime \prime}}^{\infty}(k l)$, and an IN fuzzy $O_{h^{-}}-F$ tree if $F_{O_{h}}(k l)>F_{O_{h}^{\prime \prime}}^{\infty}(k l)$.

Example 11. Consider an INGS $\breve{G}_{i}=\left(O, O_{1}, O_{2}\right)$ as shown in Figure 8. It is an $\mathrm{O}_{2}$-tree, not an $\mathrm{O}_{1}$-tree but it is IN fuzzy $O_{1}$-tree because it has an IN fuzzy-spanning subgraph $\left(O^{\prime}, O_{1}^{\prime}, O_{2}^{\prime}\right)$ as an $O_{1}^{\prime}$-tree, which is found by the deletion of $O_{1}$-edge $k_{2} k_{5}$ from $\breve{G}_{i}$. Moreover, $T_{O_{1}^{\prime}}^{\infty}\left(k_{2} k_{5}\right)=0.3>0.2=T_{O_{1}}\left(k_{2} k_{5}\right), I_{O_{1}^{\prime}}^{\infty}\left(k_{2} k_{5}\right)=0.3>0.1=$ $I_{O_{1}}\left(k_{2} k_{5}\right)$ and $F_{O_{1}^{\prime}}^{\infty}\left(k_{2} k_{5}\right)=0.4<0.5=F_{O_{1}}\left(k_{2} k_{5}\right)$.

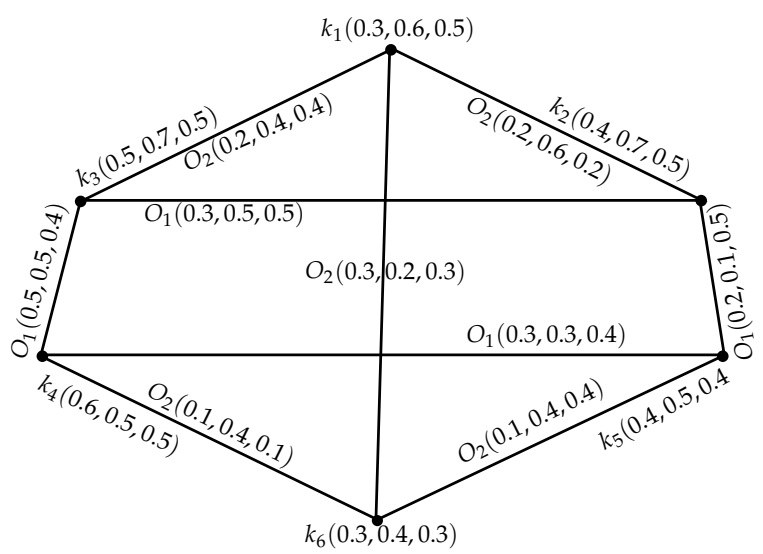

Figure 8. An IN fuzzy $O_{1}$-tree. 
Definition 22. An INGS $\check{G}_{i 1}=\left(O_{1}, O_{11}, O_{12}, \ldots, O_{1 r}\right)$ of graph structure $\check{G}_{1}=\left(P_{1}, P_{11}, P_{12}, \ldots, P_{1 r}\right)$ is said to be isomorphic to an INGS $\breve{G}_{i 2}=\left(\mathrm{O}_{2}, \mathrm{O}_{21}, \mathrm{O}_{22}, \ldots, \mathrm{O}_{2 r}\right)$ of the graph structure $\breve{G}_{2}=\left(P_{2}, P_{21}, P_{22}, \ldots, P_{2 r}\right)$, if there is a pair $(g, \psi)$, where $g: P_{1} \rightarrow P_{2}$ is a bijective mapping and $\psi$ is any permutation on this set $\{1,2, \ldots, r\}$ such that;

$$
\begin{gathered}
T_{\mathrm{O}_{1}}(k)=T_{\mathrm{O}_{2}}(g(k)), I_{\mathrm{O}_{1}}(k)=I_{O_{2}}(g(k)), F_{\mathrm{O}_{1}}(k)=F_{\mathrm{O}_{2}}(g(k)), \forall k \in P_{1}, \\
T_{\mathrm{O}_{1 h}}(k l)=T_{O_{2 \phi(h)}}(g(k) g(l)), I_{O_{1 h}}(k l)=I_{O_{2 \phi(h)}}\left(g(k) g(l), F_{Q_{1 h}}(k l)=F_{O_{2 \phi(h)}}(g(k) g(l)),\right.
\end{gathered}
$$

$\forall k l \in P_{1 h}, h=1,2, \ldots, r$.

Example 12. Let $\check{G}_{i 1}=\left(O, O_{1}, O_{2}\right)$ and $\check{G}_{i 2}=\left(O^{\prime}, O_{1}^{\prime}, O_{2}^{\prime}\right)$ be two INGSs as shown in the Figure 9.

$\check{G}_{i 1}$ and $\breve{G}_{i 2}$ are isomorphic under $(g, \psi)$, where $g: P \rightarrow P^{\prime}$ is a bijective mapping and $\psi$ is the permutation on $\{1,2\}$, which is defined as $\psi(1)=2, \psi(2)=1$, and the following conditions hold:

$$
\begin{aligned}
T_{O}\left(k_{h}\right) & =T_{O^{\prime}}\left(g\left(k_{h}\right)\right), \\
I_{O}\left(k_{h}\right) & =I_{O^{\prime}}\left(g\left(k_{h}\right)\right), \\
F_{O}\left(k_{h}\right) & =F_{O^{\prime}}\left(g\left(k_{h}\right)\right),
\end{aligned}
$$

$\forall k_{h} \in P$ and

$$
\begin{aligned}
T_{O_{h}}\left(k_{h} k_{q}\right) & =T_{O_{\psi(h)}^{\prime}}\left(g\left(k_{h}\right) g\left(k_{q}\right)\right), \\
I_{O_{h}}\left(k_{h} k_{q}\right) & =I_{O_{\psi(h)}^{\prime}}\left(g\left(k_{h}\right) g\left(k_{q}\right)\right), \\
F_{O_{h}}\left(k_{h} k_{q}\right) & =F_{O_{\psi(h)}^{\prime}}^{\prime}\left(g\left(k_{h}\right) g\left(k_{q}\right)\right),
\end{aligned}
$$

$\forall k_{h} k_{q} \in P_{h}, h=1,2$
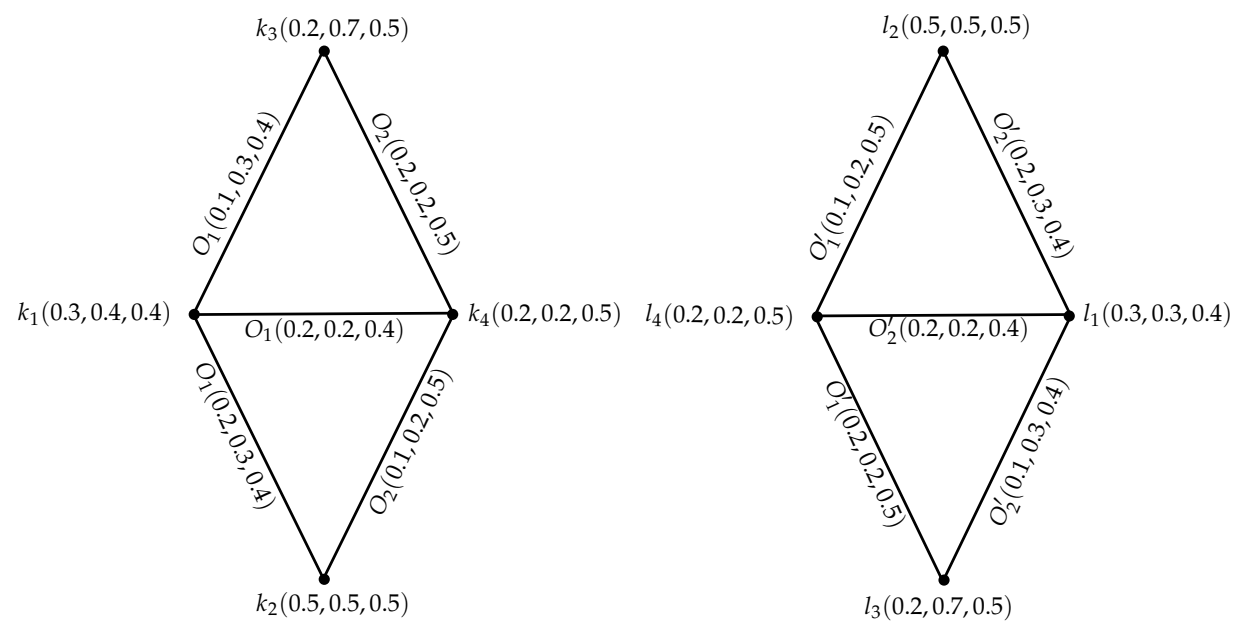

Figure 9. Two isomorphic INGSs.

Definition 23. An INGS $\check{G}_{i 1}=\left(O_{1}, O_{11}, O_{12}, \ldots, O_{1 r}\right)$ of the graph structure $\check{G}_{1}=\left(P_{1}, P_{11}, P_{12}, \ldots, P_{1 r}\right)$ is identical with an INGS $\check{G}_{i 2}=\left(\mathrm{O}_{2}, \mathrm{O}_{21}, \mathrm{O}_{22}, \ldots, \mathrm{O}_{2 r}\right)$ of the graph structure $\check{G}_{2}=\left(P_{2}, P_{21}, P_{22}, \ldots, P_{2 r}\right)$ if $g: P_{1} \rightarrow$ $P_{2}$ is a bijective mapping such that

$$
\begin{gathered}
T_{O_{1}}(k)=T_{O_{2}}(g(k)), I_{O_{1}}(k)=I_{O_{2}}(g(k)), F_{O_{1}}(k)=F_{O_{2}}(g(k)), \forall k \in P_{1}, \\
T_{O_{1 h}}(k l)=T_{O_{2 h}}(g(k) g(l)), I_{O_{1 h}}(k l)=I_{O_{2 h}}(g(k) g(l)), F_{O_{1 h}}(k l)=F_{O_{2(h)}}(g(k) g(l)),
\end{gathered}
$$


$\forall k l \in P_{1 h}, h=1,2, \ldots, r$.

Example 13. Let $\breve{G}_{i 1}=\left(O, O_{1}, O_{2}\right)$ and $\check{G}_{i 2}=\left(O^{\prime}, O_{1}^{\prime}, O_{2}^{\prime}\right)$ be two INGSs of the GSs $\breve{G}_{1}=\left(P, P_{1}, P_{2}\right), \breve{G}_{2}=$ $\left(P^{\prime}, P_{1}^{\prime}, P_{2}^{\prime}\right)$, respectively, as they are shown in Figures 10 and 11.

SVINGSs $\breve{G}_{i 1}$ and $\breve{G}_{i 2}$ are identical under $g: P \rightarrow P^{\prime}$ is defined as :

$g\left(k_{1}\right)=l_{2}, g\left(k_{2}\right)=l_{1}, g\left(k_{3}\right)=l_{4}, g\left(k_{4}\right)=l_{3}, g\left(k_{5}\right)=l_{5}, g\left(k_{6}\right)=l_{8}, g\left(k_{7}\right)=l_{7}, g\left(k_{8}\right)=l_{6}$.

Moreover, $T_{O}\left(k_{h}\right)=T_{O^{\prime}}\left(\left(k_{h}\right)\right), I_{O}\left(k_{h}\right)=I_{O^{\prime}}\left(g\left(k_{h}\right)\right), F_{O}\left(k_{h}\right)=F_{O^{\prime}}\left(g\left(k_{h}\right)\right), \forall k_{h} \in P$ and $T_{O_{h}}\left(k_{h} k_{q}\right)=$ $T_{O_{h}^{\prime}}\left(g\left(k_{h}\right) g\left(k_{q}\right)\right), I_{O_{h}}\left(k_{h} k_{q}\right)=I_{O_{h}^{\prime}}\left(g\left(k_{h}\right) g\left(k_{q}\right)\right), F_{O_{h}}\left(k_{h} k_{q}\right)=F_{O_{h}^{\prime}}\left(g\left(k_{h}\right) g\left(k_{q}\right)\right), \forall k_{h} k_{q} \in P_{h}, h=1,2$.

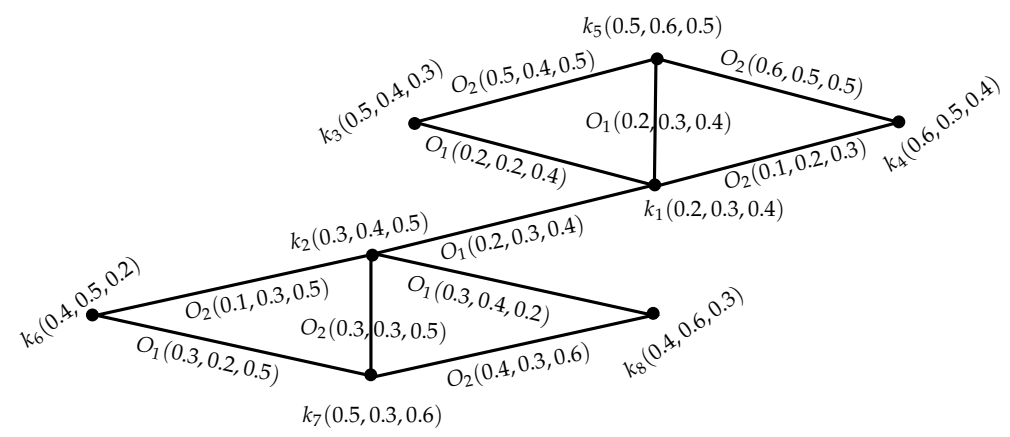

Figure 10. An INGS $\check{G}_{i 1}$.

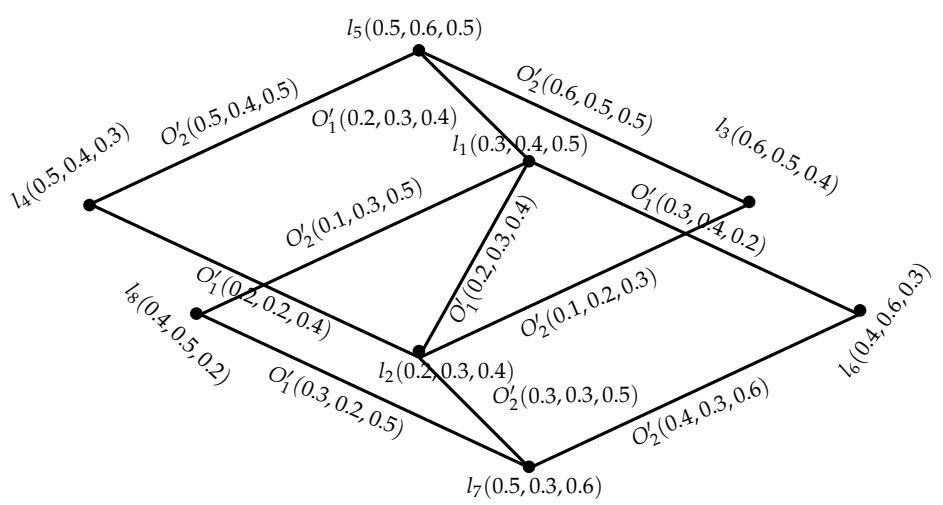

Figure 11. An INGS $\check{G}_{i 2}$.

Definition 24. Let $\breve{G}_{i}=\left(O, O_{1}, O_{2}, \ldots, O_{r}\right)$ be an INGS and $\psi$ is any permutation on $\left\{O_{1}, O_{2}, \ldots, O_{r}\right\}$ and on set $\{1,2, \ldots, r\}$, that is, $\psi\left(O_{h}\right)=O_{q}$ if and only if $\psi(h)=q \forall h$. If $k l \in O_{h}$, for any $h$ and

$T_{O_{h}^{\psi}}(k l)=T_{O}(k) \wedge T_{O}(l)-\bigvee_{q \neq h} T_{\psi\left(O_{q}\right)}(k l), I_{O_{h}^{\psi}}(k l)=I_{O}(k) \wedge I_{O}(l)-\bigvee_{q \neq h} I_{\psi\left(O_{q}\right)}(k l)$,

$F_{O_{h}^{\psi}}(k l)=F_{O}(k) \vee F_{O}(l)-\bigwedge_{q \neq h} T_{\psi\left(O_{q}\right)}(k l), h=1,2, \ldots, r$, then, $k l \in O_{t}^{\psi}$, where $t$ is chosen such that

$T_{O_{t}^{\psi}}(k l) \geq T_{O_{h}^{\psi}}(k l), I_{O_{t}^{\psi}}(k l) \geq I_{O_{h}^{\psi}}(k l), F_{O_{t}^{\psi}}(k l) \geq F_{O_{h}^{\psi}}(k l) \forall h$. In addition, INGS $\left(O, O_{1}^{\psi}, O_{2}^{\psi}, \ldots, O_{r}^{\psi}\right)$ is called a $\psi$-complement of an INGS $\breve{G}_{i}$, and it is symbolized as $\breve{G}_{i}^{\psi c}$.

Example 14. Let $O=\left\{\left(k_{1}, 0.3,0.4,0.7\right),\left(k_{2}, 0.5,0.6,0.4\right),\left(k_{3}, 0.7,0.5,0.3\right)\right\}, O_{1}=\left\{\left(k_{1} k_{3}, 0.3,0.4,0.3\right)\right\}$, $\mathrm{O}_{2}=\left\{\left(k_{2} k_{3}, 0.5,0.4,0.3\right)\right\}, O_{3}=\left\{\left(k_{1} k_{2}, 0.3,0.3,0.4\right)\right\}$ be IN subsets of $P, P_{1}, P_{2}, P_{3}$, respectively. 
Thus, $\breve{G}_{i}=\left(O, O_{1}, O_{2}, O_{3}\right)$ is an INGS of GS $\breve{G}=\left(P, P_{1}, P_{2}, P_{3}\right)$. Let $\psi\left(O_{1}\right)=O_{2}, \psi\left(O_{2}\right)=O_{3}, \psi\left(O_{3}\right)=O_{1}$, where $\psi$ is permutation on $\left\{\mathrm{O}_{1}, \mathrm{O}_{2}, \mathrm{O}_{3}\right\}$. Now, for $k_{1} k_{3}, k_{2} k_{3}, k_{1} k_{2} \in O_{1}, O_{2}, O_{3}$, respectively:

$T_{O_{1}^{\psi}}\left(k_{1} k_{3}\right)=0, I_{O_{1}^{\psi}}\left(k_{1} k_{3}\right)=0, F_{O_{1}^{\psi}}\left(k_{1} k_{3}\right)=0.7, T_{O_{2}^{\psi}}\left(k_{1} k_{3}\right)=0, I_{O_{2}^{\psi}}\left(k_{1} k_{3}\right)=0, F_{O_{2}^{\psi}}\left(k_{1} k_{3}\right)=0.7$, $T_{O_{3}^{\psi}}\left(k_{1} k_{3}\right)=0.3, I_{O_{3}^{\psi}}\left(k_{1} k_{3}\right)=0.4, F_{O_{3}^{\psi}}\left(k_{1} k_{3}\right)=0.7$. So $k_{1} k_{3} \in O_{3}^{\psi}$,

$T_{O_{1}^{\psi}}\left(k_{2} k_{3}\right)=0.5, I_{O_{1}^{\psi}}\left(k_{2} k_{3}\right)=0.5, F_{O_{1}^{\psi}}\left(k_{2} k_{3}\right)=0.4, T_{O_{2}^{\psi}}\left(k_{2} k_{3}\right)=0, I_{O_{2}^{\psi}}\left(k_{2} k_{3}\right)=0.1, F_{O_{2}^{\psi}}\left(k_{2} k_{3}\right)=0.4$, $T_{O_{3}^{\psi}}\left(k_{2} k_{3}\right)=0, I_{O_{3}^{\psi}}\left(k_{2} k_{3}\right)=0.1, F_{O_{3}^{\psi}}\left(k_{2} k_{3}\right)=0.4$. So $k_{2} k_{3} \in O_{1}^{\psi}$,

$T_{O_{1}^{\psi}}\left(k_{1} k_{2}\right)=0, I_{O_{1}^{\psi}}\left(k_{1} k_{2}\right)=0.1, F_{O_{1}^{\psi}}\left(k_{1} k_{2}\right)=0.7, T_{O_{2}^{\psi}}\left(k_{1} k_{2}\right)=0.3, I_{O_{2}^{\psi}}\left(k_{1} k_{2}\right)=0.4, F_{O_{2}^{\psi}}\left(k_{1} k_{2}\right)=0.7$, $T_{O_{3}^{\psi}}\left(k_{1} k_{2}\right)=0, I_{O_{3}^{\psi}}\left(k_{1} k_{2}\right)=0.1, F_{O_{3}^{\psi}}\left(k_{1} k_{2}\right)=0.7$. This shows $k_{1} k_{2} \in O_{2}^{\psi}$.

Hence, $\breve{G}_{i}^{\psi c}=\left(O, O_{1}^{\psi}, O_{2}^{\psi}, O_{3}^{\psi}\right)$ is a $\psi$-complement of an INGS $\breve{G}_{i}$ as presented in Figure 12.

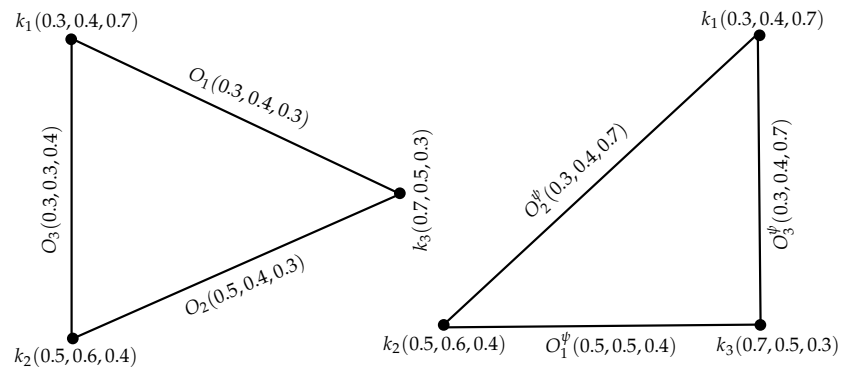

Figure 12. INGSs $\check{G}_{i}, \breve{G}_{i}^{\psi c}$.

Proposition 1. A $\psi$-complement of an INGS $\check{G}_{i}=\left(O, O_{1}, O_{2}, \ldots, O_{r}\right)$ is a strong INGS. Moreover, if $\psi(h)=t$, where $h, t \in\{1,2, \ldots, r\}$; then, all $O_{t}$-edges in an INGS $\left(O, O_{1}, O_{2}, \ldots, O_{r}\right)$ become $O_{h}^{\psi}$-edges in $\left(O, O_{1}^{\psi}, O_{2}^{\psi}, \ldots, O_{r}^{\psi}\right)$.

Proof. By definition of $\psi$-complement,

$$
\begin{aligned}
& T_{O_{h}^{\psi}}(k l)=T_{O}(k) \wedge T_{O}(l)-\bigvee_{q \neq h} T_{\psi\left(O_{q}\right)}(k l), \\
& I_{O_{h}^{\psi}}(k l)=I_{O}(k) \wedge I_{O}(l)-\bigvee_{q \neq h} I_{\psi\left(O_{q}\right)}(k l), \\
& F_{O_{h}^{\psi}}(k l)=F_{O}(k) \vee F_{O}(l)-\bigwedge_{q \neq h} F_{\psi\left(O_{q}\right)}(k l),
\end{aligned}
$$

for $h \in\{1,2, \ldots, r\}$. For Expression 1 .

As $T_{O}(k) \wedge T_{O}(l) \geq 0, \bigvee_{q \neq h} T_{\psi\left(O_{q}\right)}(k l) \geq 0$ and $T_{O_{h}}(k l) \leq T_{O}(k) \wedge T_{O}(l) \forall O_{h}$.

$\Rightarrow \bigvee_{q \neq h} T_{\psi\left(O_{q}\right)}(k l) \leq T_{O}(k) \wedge T_{O}(l) \Rightarrow T_{O}(k) \wedge T_{O}(l)-\bigvee_{q \neq h} T_{\psi\left(O_{q}\right)}(k l) \geq 0$.

Hence, $T_{O_{h}^{\psi}}(k l) \geq 0 \forall h$.

Furthermore, $T_{O_{h}^{\psi}}(k l)$ gets a maximum value, when $\underset{q \neq h}{\bigvee} T_{\psi\left(O_{q}\right)}(k l)$ is zero. Clearly, when $\psi\left(O_{h}\right)=O_{t}$ and $k l$ is an $O_{t}$-edge, then $\bigvee_{q \neq h} T_{\psi\left(O_{q}\right)}(k l)$ attains zero value. Hence,

$$
T_{O_{h}^{\psi}}(k l)=T_{O}(k) \wedge T_{O}(l), \text { for }(k l) \in O_{t}, \psi\left(O_{h}\right)=O_{t} .
$$


Similarly, for I, the results are:

Since $I_{O}(k) \wedge I_{O}(l) \geq 0, \bigvee_{q \neq h} I_{\psi\left(O_{q}\right)}(k l) \geq 0$ and $I_{O_{h}}(k l) \leq I_{O}(k) \wedge I_{O}(l) \forall O_{h}$.

$\Rightarrow \bigvee_{q \neq h} I_{\psi\left(O_{q}\right)}(k l) \leq I_{O}(k) \wedge I_{O}(l) \Rightarrow I_{O}(k) \wedge I_{O}(l)-\bigvee_{q \neq h} I_{\psi\left(O_{q}\right)}(k l) \geq 0$.

Therefore, $I_{O_{h}^{\psi}}(k l) \geq 0 \forall i$.

Value of the $I_{O_{h}^{\psi}}(k l)$ is maximum when $\bigvee_{q \neq h} I_{\psi\left(O_{q}\right)}(k l)$ gets zero value. Clearly, when $\psi\left(O_{h}\right)=O_{t}$ and $k l$ is an $O_{t}$-edge, then $\underset{q \neq h}{\bigvee} I_{\psi\left(O_{q}\right)}(k l)$ is zero. Thus,

$$
I_{O_{h}^{\psi}}(k l)=I_{O}(k) \wedge I_{O}(l), \text { for }(k l) \in O_{t}, \psi\left(O_{h}\right)=O_{t} .
$$

On a similar basis for $\mathrm{F}$ in $\psi$-complement, the results are:

Since $F_{O}(k) \vee F_{O}(l) \geq 0, \bigwedge_{q \neq h} F_{\psi\left(O_{q}\right)}(k l) \geq 0$ and $F_{O_{h}}(k l) \leq F_{O}(k) \vee F_{O}(l) \forall O_{h}$.

$\Rightarrow \bigwedge_{q \neq h} F_{\psi\left(O_{q}\right)}(k l) \leq F_{O}(k) \vee F_{O}(l) \Rightarrow F_{O}(k) \vee F_{O}(l)-\bigwedge_{q \neq h} F_{\psi\left(O_{q}\right)}(k l) \geq 0$.

Hence, $F_{O_{h}^{\psi}}(k l) \geq 0 \forall h$.

Furthermore, $F_{O_{h}^{\psi}}(k l)$ is maximum, when $\bigwedge_{q \neq h} F_{\psi\left(O_{q}\right)}(k l)$ is zero. Definitely, when $\psi\left(O_{h}\right)=O_{t}$ and $k l$ is an $O_{t}$-edge, then $\bigwedge_{q \neq h} F_{\psi\left(O_{q}\right)}(k l)$ is zero. Hence,

$$
F_{O_{h}^{\psi}}(k l)=F_{O}(k) \vee F_{O}(l), \text { for }(k l) \in O_{t}, \psi\left(O_{h}\right)=O_{t} .
$$

Expressions (4)-(6) give the required proof.

Definition 25. Let $\breve{G}_{i}=\left(O, O_{1}, O_{2}, \ldots, O_{r}\right)$ be an INGS and $\psi$ be any permutation on $\{1,2, \ldots, r\}$. Then,

(i) $\breve{G}_{i}$ is a self-complementary INGS if $\breve{G}_{i}$ is isomorphic to $\breve{G}_{i}^{\psi c}$;

(ii) $\breve{G}_{i}$ is a strong self-complementary INGS if $\breve{G}_{i}$ is identical to $\breve{G}_{i}^{\psi c}$.

Definition 26. Let $\breve{G}_{i}=\left(O, O_{1}, O_{2}, \ldots, O_{r}\right)$ be an INGS. Then,

(i) $\breve{G}_{i}$ is a totally self-complementary INGS if $\breve{G}_{i}$ is isomorphic to $\breve{G}_{i}^{\psi c}, \forall$ permutations $\psi$ on $\{1,2, \ldots, r\}$;

(ii) $\breve{G}_{i}$ is a totally-strong self-complementary INGS if $\breve{G}_{i}$ is identical to $\breve{G}_{i}^{\psi c}, \forall$ permutations $\psi$ on $\{1,2, \ldots, r\}$.

Example 15. INGS $\breve{G}_{i}=\left(O, O_{1}, O_{2}, O_{3}\right)$ in Figure 13 is totally-strong self-complementary INGS.

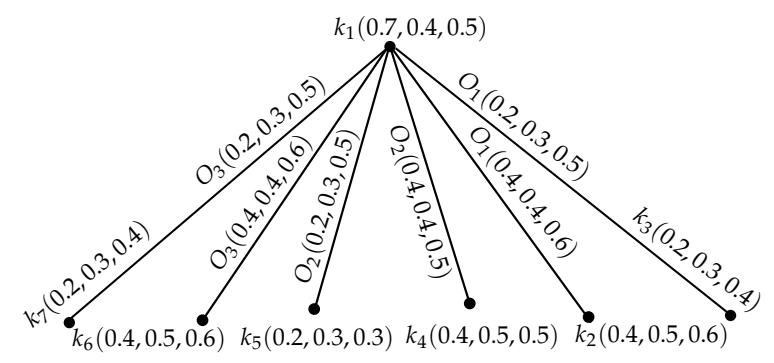

Figure 13. Totally-strong self-complementary INGS.

Theorem 1. A strong INGS is a totally self-complementary INGS and vice versa. 
Proof. Consider any strong INGS $\breve{G}_{i}$ and Permutation $\psi$ on $\{1,2, \ldots, \mathrm{r}\}$. By proposition $1, \psi$-complement of an INGS $\breve{G}_{i}=\left(O, O_{1}, O_{2}, \ldots, O_{r}\right)$ is a strong INGS. Moreover, if $\psi^{-1}(t)=h$, where $h, t \in\{1,2, \ldots, r\}$, then all $O_{t}$-edges in an INGS $\left(O, O_{1}, O_{2}, \ldots, O_{r}\right)$ become $O_{h}^{\psi}$-edges in $\left(O, O_{1}^{\psi}, O_{2}^{\psi}, \ldots, O_{r}^{\psi}\right)$, this leads

$$
\begin{aligned}
T_{O_{t}}(k l)=T_{O}(k) \wedge T_{O}(l) & =T_{O_{h}^{\psi}}(k l), I_{O_{t}}(k l)=I_{O}(k) \wedge I_{O}(l)=I_{O_{h}^{\psi}}(k l), \\
F_{O_{t}}(k l) & =F_{O}(k) \vee F_{O}(l)=F_{O_{h}^{\psi}}(k l) .
\end{aligned}
$$

Therefore, under $g: P \rightarrow P$ (identity mapping), $\breve{G}_{i}$ and $\breve{G}_{i}^{\psi}$ are isomorphic, such that

$$
T_{O}(k)=T_{O}(g(k)), I_{O}(k)=I_{O}(g(k)), F_{O}(k)=F_{O}(g(k))
$$

and

$$
\begin{gathered}
T_{O_{t}}(k l)=T_{O_{h}^{\psi}}(g(k) g(l))=T_{O_{h}^{\psi}}(k l), \\
I_{O_{t}}(k l)=I_{O_{h}^{\psi}}(g(k) g(l))=I_{O_{h}^{\psi}}(k l), \\
F_{O_{t}}(k l)=F_{O_{h}^{\psi}}(g(k) g(l))=F_{O_{h}^{\psi}}(k l),
\end{gathered}
$$

$\forall k l \in P_{t}$, for $\psi^{-1}(t)=h ; \mathrm{h}, \mathrm{t}=1,2, \ldots, r$.

For each permutation $\psi$ on $\{1,2, \ldots, r\}$, this holds. Hence, $\breve{G}_{i}$ is a totally self-complementary INGS.

Conversely, let $\breve{G}_{i}$ is isomorphic to $\breve{G}_{i}^{\psi}$ for each permutation $\psi$ on $\{1,2, \ldots, r\}$. Then, by definitions of $\psi$-complement of INGS and isomorphism of INGS, we have

$$
\begin{gathered}
T_{O_{t}}(k l)=T_{O_{h}^{\psi}}(g(k) g(l))=T_{O}(g(k)) \wedge T_{O}(g(l))=T_{O}(k) \wedge T_{O}(l), \\
I_{O_{t}}(k l)=I_{O_{h}^{\psi}}(g(k) g(l))=I_{O}(g(k)) \wedge I_{O}(g(l))=T_{O}(k) \wedge I_{O}(l), \\
F_{O_{t}}(k l)=F_{O_{h}^{\psi}}(g(k) g(l))=F_{O}(g(k)) \vee F_{O}(g(l))=F_{O}(k) \vee F_{O}(l),
\end{gathered}
$$

$\forall k l \in P_{t}, \mathrm{t}=1,2, \ldots, \mathrm{r}$. Hence, $\check{G}_{i}$ is strong INGS.

Remark 1. Each self-complementary INGS is a totally self-complementary INGS.

Theorem 2. If $\breve{G}=\left(P, P_{1}, P_{2}, \ldots, P_{r}\right)$ is a totally strong self-complementary $G S$ and $O=\left(T_{O}, I_{O}, F_{O}\right)$ is an IN subset of $P$, where $T_{O}, I_{O}, F_{O}$ are the constant functions, then any strong INGS of $\breve{G}$ with IN vertex set $O$ is necessarily totally-strong self-complementary INGS.

Proof. Let $u \in[0,1], v \in[0,1]$ and $w \in[0,1]$ be three constants, and

$$
T_{O}(k)=u, I_{O}(k)=v, F_{O}(k)=w \forall k \in P .
$$

Since $\breve{G}$ is a totally strong self-complementary GS, so, for each permutation $\psi^{-1}$ on $\{1,2, \ldots, r\}$, there exists a bijective mapping $g: P \rightarrow P$, such that, for each $P_{t}$-edge $(k l),(g(k) g(l))\left[a P_{h}\right.$-edge in $\breve{G}$ ] is a $P_{t}$-edge in $\breve{G}^{\psi^{-1} c}$. Thus, for every $O_{t}$-edge $(k l),(\mathrm{g}(\mathrm{k}) \mathrm{g}(\mathrm{l}))$ [an $O_{h}$-edge in $\left.\check{G}_{i}\right]$ is an $O_{t}^{\psi}$-edge in $\check{G}_{i}{ }^{\psi^{-1} c}$. Moreover, $\check{G}_{i}$ is a strong INGS, so

$$
T_{O}(k)=u=T_{O}(g(k)), I_{O}(k)=v=I_{O}(g(k)), F_{O}(k)=w=F_{O}(g(k)) \forall k \in P
$$

and 


$$
\begin{gathered}
T_{O_{t}}(k l)=T_{O}(k) \wedge T_{O}(l)=T_{O}(g(k)) \wedge T_{O}(g(l))=T_{O_{h}^{\psi}}(g(k) g(l)), \\
I_{O_{t}}(k l)=I_{O}(k) \wedge I_{O}(l)=I_{O}(g(k)) \wedge I_{O}(g(l))=I_{O_{h}^{\psi}}(g(k) g(l)), \\
F_{O_{t}}(k l)=F_{O}(k) \vee I_{O}(l)=F_{O}(g(k)) \vee F_{O}(g(l))=F_{O_{h}^{\psi}}(g(k) g(l)),
\end{gathered}
$$

$\forall k l \in P_{h}, h=1,2, \ldots, r$.

This shows that $\breve{G}_{i}$ is a strong self-complementary INGS. This exists for each permutation $\psi$ and $\psi^{-1}$ on set $\{1,2, \ldots, r\}$, thus $\breve{G}_{i}$ is a totally strong self-complementary INGS. Hence, required proof is obtained.

Remark 2. Converse of the Theorem 2 may or may not true, as an INGS shown in Figure 2 is totally strong self-complementary INGS, and it is also a strong INGS with a totally strong self-complementary underlying GS but $T_{O}, I_{O}, F_{O}$ are not the constant-valued functions.

\section{Application}

First, we explain the general procedure of this application by the following algorithm.

\section{Algorithm: Crucial interdependence relations}

Step 1. Input vertex set $P=\left\{B_{1}, B_{2}, \ldots, B_{n}\right\}$ and IN set $O$ defined on $P$.

Step 2. Input IN set of interdependence relations of any vertex with all other vertices and calculate $T, F$, and $I$ of every pair of vertices by using, $T\left(B_{i} B_{j}\right) \leq \min \left(T\left(B_{i}\right), T\left(B_{j}\right)\right), F\left(B_{i} B_{j}\right) \leq$ $\max \left(F\left(B_{i}\right), F\left(B_{j}\right)\right), I\left(B_{i} B_{j}\right) \leq \min \left(I\left(B_{i}\right), I\left(B_{j}\right)\right)$.

Step 3. Repeat the Step 2 for every vertex in $P$.

Step 4. Define relations $P_{1}, P_{2}, \ldots, P_{n}$ on set $P$ such that $\left(P, P_{1}, P_{2}, \ldots, P_{n}\right)$ is a GS.

Step 5. Consider an element of that relation, for which its value of $T$ is comparatively high, and its values of $F$ and $I$ are lower than other relations.

Step 6. Write down all elements in relations with $T, F$ and $I$ values, corresponding relations $\mathrm{O}_{1}, \mathrm{O}_{2}, \ldots, \mathrm{O}_{n}$ are IN sets on $P_{1}, P_{2}, P_{3}, \ldots, P_{n}$, respectively, and $\left(O, \mathrm{O}_{1}, \mathrm{O}_{2}, \ldots, \mathrm{O}_{n}\right)$ is an INGS.

Human beings, the main creatures in the world, depend on many things for their survival. Interdependence is a very important relationship in the world. It is a natural phenomenon that nobody can be $100 \%$ independent, and the whole world is relying on interdependent relationships. Provinces or states of any country, especially of a progressive country, can not be totally independent, more or less they have to depend on each other. They depend on each other for many things, that is, there are many interdependent relationships among provinces or states of a progressive country-for example, education, natural energy resources, agricultural items, industrial products, and water resources, etc. However, all of these interdependent relationships are not of equal importance. Some are very important to run the system of a progressive country. Between any two provinces, all interdependent relationships do not have the same strength. Some interdependent relationships are like the backbone for the country. We can make an INGS of provinces or states of a progressive country, and can highlight those interdependent relationships, due to which the system of the country is running properly. This INGS can guide the government as to which interdependent relationships are very crucial, and they must try to make them strong and overcome the factors destroying or weakening them.

We consider a set $P$ of provinces and states of Pakistan:

$P=\{$ Punjab, Sindh, Khyber Pakhtunkhawa(KPK), Balochistan, Gilgit-Baltistan, Azad Jammu and Kashmir(AJK) \}. Let $O$ be the IN set on $P$, as defined in Table 1 . 
Table 1. IN set $\mathrm{O}$ of provinces of Pakistan.

\begin{tabular}{cccc}
\hline Provinces or States & T & I & F \\
\hline Punjab & 0.5 & 0.3 & 0.3 \\
Sindh & 0.5 & 0.4 & 0.4 \\
Khyber Pakhtunkhawa(KPK) & 0.4 & 0.4 & 0.4 \\
Balochistan & 0.3 & 0.4 & 0.4 \\
Gilgit-Baltistan & 0.3 & 0.4 & 0.4 \\
Azad Jammu and Kashmir & 0.3 & 0.4 & 0.3 \\
\hline
\end{tabular}

In Table 1 , symbol $T$ demonstrates the positive role of that province or state for the strength of the Federal Government, and symbol $F$ indicates its negative role, whereas $I$ denotes the percentage of ambiguity of its role for the strength of the Federal Government. Let us use the following alphabets for the provinces' names:

PU = Punjab, SI = Sindh, KPK = Khyber Pakhtunkhwa, BA = Balochistan, GB = Gilgit-Baltistan, AJK $=$ Azad Jammu and Kashmir. For every pair of provinces of Pakistan in set $P$, different interdependent relationships with their $T, I$ and $F$ values are demonstrated in Tables 2-6.

Table 2. IN set of interdependent relations between Punjab and other provinces.

\begin{tabular}{cccc}
\hline Type of Interdependent Relationships & (PU, SI) & (PU, KPK) & (PU, BA) \\
\hline Education & $(0.5,0.1,0.1)$ & $(0.4,0.3,0.2)$ & $(0.3,0.2,0.2)$ \\
Natural energy resources & $(0.3,0.2,0.3)$ & $(0.4,0.2,0.2)$ & $(0.3,0.2,0.1)$ \\
Agricultural items & $(0.3,0.2,0.2)$ & $(0.4,0.2,0.1)$ & $(0.3,0.2,0.1)$ \\
Industrial products & $(0.4,0.2,0.1)$ & $(0.4,0.1,0.1)$ & $(0.3,0.1,0.1)$ \\
Water resources & $(0.3,0.1,0.1)$ & $(0.4,0.3,0.2)$ & $(0.2,0.2,0.2)$ \\
\hline
\end{tabular}

Table 3. IN set of interdependent relationships between Sindh and other provinces.

\begin{tabular}{cccc}
\hline Type of Interdependent Relationships & (SI, KPK) & (SI, BA) & (SI, GB) \\
\hline Education & $(0.3,0.2,0.1)$ & $(0.3,0.2,0.3)$ & $(0.3,0.2,0.4)$ \\
Natural energy resources & $(0.3,0.2,0.3)$ & $(0.3,0.1,0.0)$ & $(0.2,0.2,0.4)$ \\
Agricultural items & $(0.4,0.1,0.1)$ & $(0.3,0.1,0.2)$ & $(0.3,0.1,0.1)$ \\
Industrial products & $(0.4,0.2,0.1)$ & $(0.3,0.2,0.2)$ & $(0.3,0.2,0.2)$ \\
Water resources & $(0.3,0.2,0.2)$ & $(0.2,0.3,0.2)$ & $(0.2,0.2,0.3)$ \\
\hline
\end{tabular}

Table 4. IN set of interdependent relationships between KPK and other provinces.

\begin{tabular}{cccc}
\hline Type of Interdependent Relationships & $\mathbf{( K P K , ~ B A ) ~}$ & $\mathbf{( K P K , ~ G B )}$ & $\mathbf{( K P K , ~ A J K ) ~}$ \\
\hline Education & $(0.1,0.4,0.3)$ & $(0.1,0.4,0.3)$ & $(0.1,0.4,0.4)$ \\
Natural energy resources & $(0.3,0.2,0.1)$ & $(0.3,0.2,0.2)$ & $(0.3,0.3,0.2)$ \\
Agricultural items & $(0.1,0.2,0.4)$ & $(0.1,0.4,0.4)$ & $(0.1,0.3,0.3)$ \\
Industrial products & $(0.1,0.3,0.4)$ & $(0.1,0.4,0.3)$ & $(0.1,0.2,0.2)$ \\
Water resources & $(0.3,0.2,0.2)$ & $(0.3,0.3,0.2)$ & $(0.3,0.2,0.2)$ \\
\hline
\end{tabular}


Table 5. IN set of interdependent relationships between AJK and other provinces.

\begin{tabular}{cccc}
\hline Type of Interdependent Relationships & (AJK, PU) & (AJK, SI) & (AJK, BA) \\
\hline Education & $(0.3,0.1,0.1)$ & $(0.1,0.4,0.3)$ & $(0.1,0.3,0.4)$ \\
Natural energy resources & $(0.1,0.2,0.3)$ & $(0.2,0.4,0.3)$ & $(0.3,0.3,0.3)$ \\
Agricultural items & $(0.3,0.2,0.1)$ & $(0.3,0.3,0.2)$ & $(0.3,0.2,0.2)$ \\
Industrial products & $(0.3,0.2,0.2)$ & $(0.3,0.2,0.2)$ & $(0.3,0.2,0.3)$ \\
Water resources & $(0.3,0.2,0.1)$ & $(0.3,0.3,0.2)$ & $(0.3,0.0,0.1)$ \\
\hline
\end{tabular}

Table 6. IN set of interdependent relationships of Gilgit-Baltistan with other provinces.

\begin{tabular}{cccc}
\hline Type of Interdependent Relationships & $\mathbf{( G B}, \mathbf{P U})$ & $\mathbf{( G B}, \mathbf{B A})$ & $\mathbf{( G B}, \mathbf{A J K})$ \\
\hline Education & $(0.3,0.2,0.1)$ & $(0.1,0.4,0.4)$ & $(0.2,0.1,0.4)$ \\
Natural energy resources & $(0.1,0.3,0.4)$ & $(0.3,0.1,0.0)$ & $(0.2,0.2,0.4)$ \\
Agricultural items & $(0.3,0.2,0.2)$ & $(0.1,0.3,0.3)$ & $(0.1,0.4,0.4)$ \\
Industrial products & $(0.3,0.3,0.2)$ & $(0.2,0.4,0.4)$ & $(0.1,0.4,0.2)$ \\
Water resources & $(0.2,0.3,0.3)$ & $(0.2,0.3,0.2)$ & $(0.3,0.1,0.1)$ \\
\hline
\end{tabular}

Many relations can be defined on the set $P$, we define following relations on set $P$ as:

$P_{1}=$ Education, $P_{2}=$ Natural energy resources,$P_{3}=$ Agricultural items, $P_{4}=$ Industrial products, $P_{5}=$ Water resources, such that $\left(P, P_{1}, P_{2}, P_{3}, P_{4}, P_{5}\right)$ is a GS. Any element of a relation demonstrates a particular interdependent relationship between these two provinces. As $\left(P, P_{1}, P_{2}, P_{3}, P_{4}, P_{5}\right)$ is GS; this is why any element can appear in only one relation. Therefore, any element will be considered in that relationship, whose value of $\mathrm{T}$ is high, and values of I, F are comparatively low, using the data of above tables.

Write down T, I and F values of the elements in relations according to the above data, such that $O_{1}$, $\mathrm{O}_{2}, \mathrm{O}_{3}, \mathrm{O}_{4}, \mathrm{O}_{5}$ are IN sets on relations $P_{1}, P_{2}, P_{3}, P_{4}, P_{5}$, respectively.

Let $P_{1}=\{($ Punjab, Sindh), (Gilgit - Baltistan, Punjab), (AzadJammuandKashmir, Punjab $)\}$;

$P_{2}=\{($ Sindh, Balochistan), (Khyber Pakhtunkhawa, Balochistan), (Balochistan, Gilgit-Baltistan), (Khyber Pakhtunkhawa, Gilgit-Baltistan)\};

$P_{3}=\{($ Sindh, Khyber Pakhtunkhwa), (Gilgit-Baltistan, Sindh) $\}$;

$P_{4}=\{($ Punjab, KhyberPakhtunkhwa), (Sindh, AzadJammuandKashmir $),($ Balochistan, Punjab) $\} ;$

$P_{5}=\{($ KheberPakhtunkhwa, AzadJammuandKashmir $),($ Balochistan, AzadJammuandKashmir $)$,

(Gilgit - Baltistan, Azad Jammu and Kashmir)\}.

Let $O_{1}=\{((P U, S I), 0.5,0.1,0.1),((G B, P U), 0.3,0.2,0.1),((A J K, P U), 0.3,0.1,0.1)\}$, $\mathrm{O}_{2}=\{((S I, B A), 0.3,0.1,0.0),((K P K, B A), 0.3,0.2,0.1),((B A, G B), 0.3,0.1,0.0)$,

$((K P K, G B), 0.3,0.2,0.2)\}$,

$\mathrm{O}_{3}=\{((S I, K P K), 0.4,0.1,0.1),((G B, S I), 0.3,0.1,0.1)\}$,

$\mathrm{O}_{4}=\{((P U, K P K), 0.4,0.1,0.1),((S I, A J K), 0.3,0.2,0.2),((B A, P U), 0.3,0.1,0.1)\}$,

$\mathrm{O}_{5}=\{((K P K, A J K), 0.3,0.2,0.2),((B A, A J K), 0.3,0.0,0.1),((G B, A J K), 0.3,0.1,0.1)\}$.

Obviously, $\left(\mathrm{O}, \mathrm{O}_{1}, \mathrm{O}_{2}, \mathrm{O}_{3}, \mathrm{O}_{4}, \mathrm{O}_{5}\right)$ is an INGS as shown in Figure 14. 


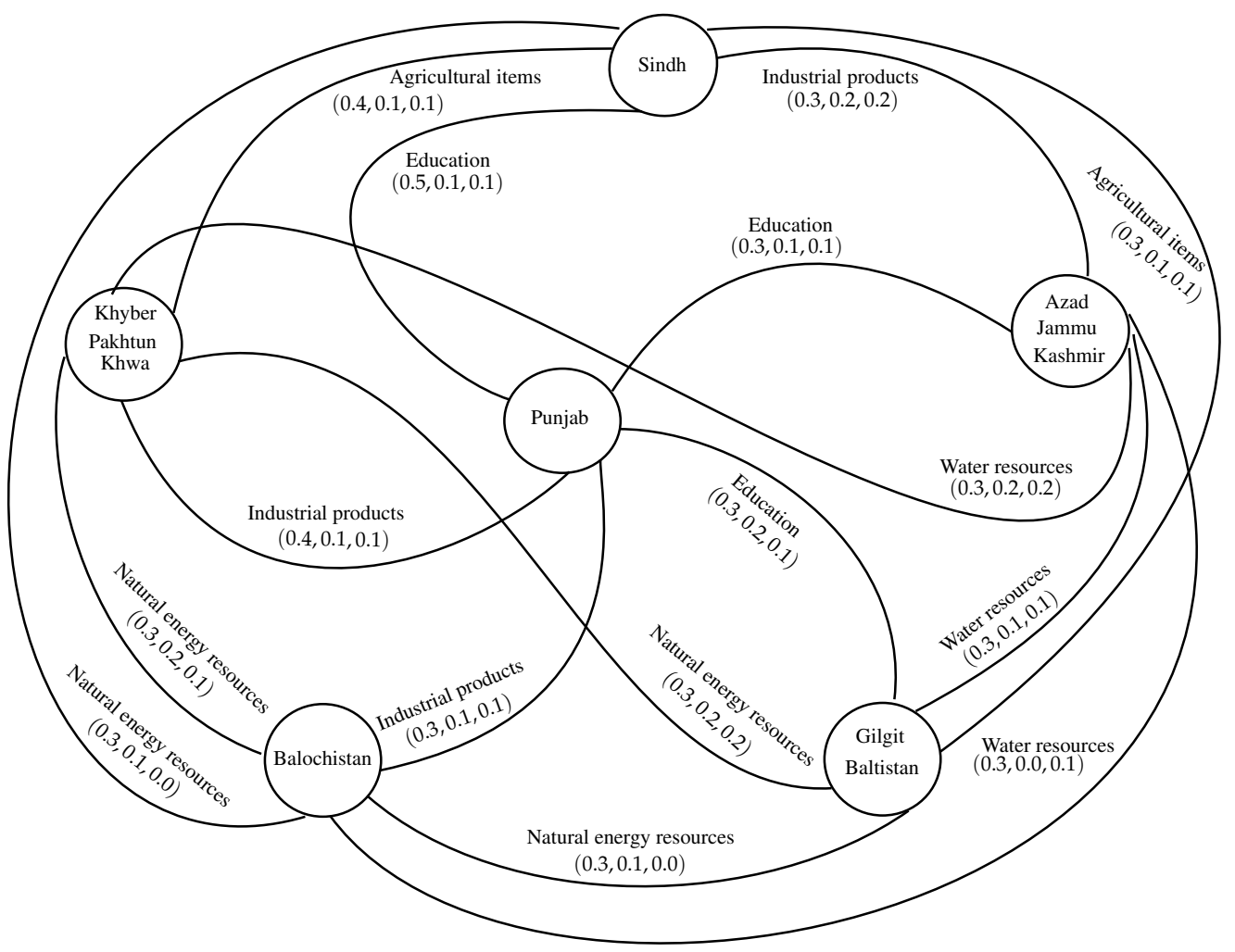

Figure 14. INGS identifying crucial interdependence relation between any two provinces.

Every edge of this INGS demonstrates the most dominating interdependent relationship between those two provinces-for example, the most dominating interdependent relationship between Punjab and Gilgit-Baltistan is education, and its T, F and I values are 0.3, 0.2 and 0.1 , respectively. It shows that education is the strongest connection bond between Punjab and Gilgit-Baltistan; it is $30 \%$ stable, $10 \%$ unstable, and $20 \%$ unpredictable or uncertain. Using INGS, we can also elaborate the strength of any province, e.g., Punjab has the highest vertex degree for interdependent relationship education, and Balochistan has the highest vertex degree for the interdependent relationship natural energy resources. This shows that the strength of Punjab is education, and the strength of Balochistan is the natural energy resources. This INGS can be very helpful for Provincial Governments, and they can easily estimate which kind of interdependent relationships they have with other provinces, and what is the percentage of its stability and instability. It can also guide the Federal Government in regards to, between any two provinces, which relationships are crucial and what is their status. The Federal Government should be conscious of making decisions such that the most crucial interdependent relationships of its provinces are not disturbed and need to overcome the counter forces that are trying to destroy them.

\section{Conclusions}

Graph theory is a useful tool for solving combinatorial problems of different fields, including optimization, algebra, computer science, topology and operations research. An intuitionistic neutrosophic set constitutes a generalization of an intuitionistic fuzzy set. In this research paper, we have introduced the notion of intuitionistic neutrosophic graph structure. We have discussed a real-life 
application of intuitionistic neutrosophic graph structure in decision-making. Our aim is to extend our research work to (1) fuzzy rough graph structures; (2) rough fuzzy graph structures; (3) soft rough graph structures; and (4) roughness in graph structures.

Acknowledgments: The authors are thankful to the referees for their invaluable suggestions.

Author Contributions: Muhammad Akram and Muzzamal Sitara conceived and designed the experiments; Muhammad Akram performed the experiments; Muzzamal Sitara analyzed the data and wrote the paper.

Conflicts of Interest: The authors declare that they have no conflict of interest.

\section{References}

1. Zadeh, L.A. Fuzzy sets. Inf. Control 1965, 8, 338-353.

2. Atanassov, K. Intuitionistic fuzzy sets. Fuzzy Sets Syst. 1986, 20, 87-96.

3. Smarandache, F. Neutrosophy Neutrosophic Probability, Set, and Logic; Amer Res Press: Rehoboth, DE, USA, 1998.

4. Wang, H.; Smarandache, F.; Zhang, Y.Q.; Sunderraman, R. Single-valued neutrosophic sets. Multispace Multistruct. 2010, 4, 410-413.

5. Ye, J. Multicriteria decision-making method using the correlation coefficient under single-valued neutrosophic environment. Int. J. Gen. Syst. 2013, 42, 386-394.

6. Ye, J. Single-valued neutrosophic minimum spanning tree and its clustering method. J. Intell. Syst. 2014, 23, 311-324.

7. Ye, J. Improved correlation coefficients of single-valued neutrosophic sets and interval neutrosophic sets for multiple attribute decision making. J. Intell. Fuzzy Syst. 2014, 27, 2453-2462.

8. Ye, J. A multicriteria decision-making method using aggregation operators for simplified neutrosophic sets. J. Intell. Fuzzy Syst. 2014, 26, 2459-2466.

9. Bhowmik, M.; Pal, M. Intuitionistic neutrosophic set. J. Inf. Comput. Sci. 2009, 4, 142-152.

10. Bhowmik, M.; Pal, M. Intuitionistic neutrosophic set relations and some of its properties. J. Inf. Comput. Sci. 2010, 5, 183-192.

11. Kauffman, A. Introduction a la Theorie des Sous-Emsembles Flous, Masson et Cie: Renens, Switzerland; 1973; Volume 1.

12. Zadeh, L.A. Similarity relations and fuzzy orderings. Inf. Sci. 1971, 3, 177-200.

13. Rosenfeld, A. Fuzzy Graphs, Fuzzy Sets and Their Applications; Zadeh, L.A., Fu, K.S., Shimura, M., Eds.; Academic Press: New York, NY, USA, 1975; pp. 77-95.

14. Bhattacharya, P. Some remarks on fuzzy graphs. Pattern Recognit. Lett. 1987, 6, 297-302.

15. Sunitha, M.S.; Vijayakumar, A. Complement of a fuzzy graph. Indian J. Pure Appl. Math. 2002, 33, 1451-1464.

16. Bhutani, K.R.; Rosenfeld, A. Strong arcs in fuzzy graphs. Inf. Sci. 2003, 152, 319-326.

17. Parvathi, R.; Karunambigai, M.G.; Atanassov, K.T. Operations on intuitionistic fuzzy graphs. In Proceedings of the 2009 IEEE International Conference on Fuzzy Systems, Jeju Island, Korea, 20-24 August 2009; pp. 1396-1401.

18. Akram, M.; Shahzadi, S. Neutrosophic soft graphs with application. J. Intell. Fuzzy Syst. 2016, 32, 841-858.

19. Dinesh, T.; Ramakrishnan, T.V. On generalised fuzzy graph structures. Appl. Math. Sci. 2011, 5, 173-180.

20. Akram, M.; Akmal, R. Application of bipolar fuzzy sets in graph structures. Appl. Comput. Intell. Soft Comput. 2016, doi:10.1155/2016/5859080.

21. Akram, M. Sitara, M. Application of intuitionistic neutrosophic graph structures in decision-making. Ann. Fuzzy Math. Inform. 2017, 14, 1-27.

22. Akram, M.; Sitara, M. Interval-valued neutrosophic graph structures. Punjab Univ. J. Math. 2018, 50, 113-136.

23. Akram, M.; Sitara, M.; Smarandache, F. Graph structures in bipolar neutrosophic environment. Mathematics 2017, 5, 60, doi:10.3390/math5040060.

24. Akram, M.; Sitara, M. Single-Valued Neutrosophic Graph Structures. Available online: http:/ / fs.gallup.unm.edu/SingleValuedNeutrosophic.pdf (accessed on 20 November 2017). 
25. Akram, M.; Sitara, M. Novel applications of single-valued neutrosophic graph structures in decision-making. J. Appl. Math. Comput. 2017, doi:10.1007/s12190-017-1084-5.

26. Akram, M.; Sitara, M. Representation of graph Structure based on I-V neutrosophic sets. Int. J. Algebra Stat. 2017, 6, 56-80.

27. Akram, M.; Sitara, M. Bipolar neutrosophic graph structures. J. Indones. Math. Soc. 2017, 23, 55-76.

28. Akram, M.; Al-Shehrie, N.O. Intuitionistic fuzzy cycles and intuitionistic fuzzy trees. Sci. World J. 2014, doi:10.1155/2014/305836.

29. Akram, M.; Davvaz, B. Strong intuitionistic fuzzy graphs. Filomat 2012, 26, 177-196.

30. Akram, M.; Shahzadi, G. Operations on single-valued neutrosophic graphs. J. Uncertain Syst. 2017, 11, 176-196.

31. Dhavaseelan, R.; Vikramaprasad, R.; Krishnaraj, V. Certain types of neutrosophic graphs. Int J. Math. Sci. Appl. 2015, 5, 333-339.

32. Dinesh, T. A Study on Graph Structures, Incidence Algebras and Their Fuzzy Analogues. Ph.D. Thesis, Kannur University, Kannur, India, 2011.

33. Mordeson, J.N.; Nair, P.S. Fuzzy Graphs and Fuzzy Hypergraphs, 2nd ed.; Physica Verlag: Berlin/Heidelberg, Germany, 2001.

34. Smarandache, F. A Unifying Field in Logics. Neutrosophy: Neutrosophic Probability, Set and Logic; American Research Press: Rehoboth, DE, USA, 1999.

35. Tian, Z.-P.; Zhang, H.-Y.; Wang, J.; Wang, J.-Q.; Chen, X.-H. Multi-criteria decision-making method based on a cross-entropy with interval neutrosophic sets. Int. J. Syst. Sci. 2016, 47, 3598-3608.

36. Zavadskas, E.K.; Bausys, R.; Kaklauskas A.; Ubarte, I.; Kuzminske, A.; Gudiene, N. Sustainable market valuation of buildings by the singlevalued neutrosophic MAMVA method. Appl. Soft Comput. 2017, 57, 74-87.

37. Zavadskas, E.K.; Bausys, R.; Juodagalviene, B. GarnyteSapranaviciene I. Model for residential house element and material selection by neutrosophic multimoora method. Eng. Appl. Artif. Intell. 2017, 64, 315-324.

38. Bausys, R.; Juodagalviene, B. Garage location selection for residential house by WASPAS-SVNS method. J. Civil Eng. Manag. 2017, 23, 421-429.

39. Xindong, P.; Chong. L. Algorithms for neutrosophic soft decision making based on EDAS, new similarity measure and level soft set. J. Intell. Fuzzy Syst. 2017, 32, 955-968. doi: 10.3233/JIFS-161548

40. Pouresmaeil, H.; Shivanian, E.; Khorram, E.; Fathabadi, H.S. An extended method using topsis and vikor for multiple attribute decision making with multiple decision makers and single valued neutrosophic numbers. Adv. Appl. Stat. 2017, 50, 261-292.

41. Tian, Z.-P.; Wang, J.-Q.; Zhang, H.-Y. Hybrid single-valued neutrosophic MCGDM with QFD for market segment evaluation and selection. J. Intell. Fuzzy Syst. 2017, doi:10.3233/JIFS-171055.

42. Wang, J.; Zhang, X.; Zhang, H. Hotel recommendation approach based on the online consumer reviews using interval neutrosophic linguistic numbers. J. Intell. Fuzzy Syst. 2017, doi:10.3233/JIFS-171421.

43. Liang, R.; Wang, J.; Zhang, H. Evaluation of e-commerce websites: An integrated approach under a single-valued trapezoidal neutrosophic environment. Knowl.-Based Syst. 2017, 135, 44-59.

44. Nie, R.-X.; Wang, J.-Q.; Zhang, H.-Y. Solving solar-wind power station location problem using an extended WASPAS technique with Interval neutrosophic sets. Symmetry 2017, 9, 106, doi:10.3390/sym9070106.

45. Luo, S.-Z.; Cheng, P.-F.; Wang, J.-Q, Huang, Y.-J. Selecting Project Delivery Systems Based on Simplified Neutrosophic Linguistic Preference Relations. Symmetry 2017, 9, 151, doi:10.3390/sym9070151.

46. Sampathkumar, E. Generalized graph structures. Bull. Kerala Math. Assoc. 2006, 3, 65-123.

47. Mondal, T.K.; Samanta, S.K. Generalized intuitionistic fuzzy sets. J. Fuzzy Math. 2002, 10, 839-862.

(C) 2017 by the authors. Licensee MDPI, Basel, Switzerland. This article is an open access article distributed under the terms and conditions of the Creative Commons Attribution (CC BY) license (http:/ / creativecommons.org/licenses/by/4.0/). 